\title{
CCD photometric search for peculiar stars in open clusters
}

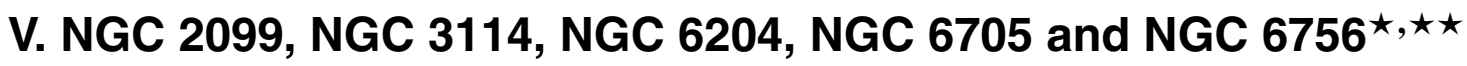

\author{
E. Paunzen ${ }^{1,2}$, O. I. Pintado ${ }^{3, \star \star \star}$, and H. M. Maitzen ${ }^{1}$
}

\author{
${ }^{1}$ Institut für Astronomie der Universität Wien, Türkenschanzstr. 17, 1180 Wien, Austria \\ 2 Zentraler Informatikdienst der Universität Wien, Universitätsstr. 7, 1010 Wien, Austria \\ 3 Departamento de Física, Facultad de Ciencias Exactas y Tecnología, Universidad Nacional de Tucumán, Argentina - Consejo \\ Nacional de Investigaciones Científicas y Técnicas de la República Argentina
}

Received 7 February 2003 / Accepted 16 June 2003

\begin{abstract}
We have investigated 1008 objects in the area of five intermediate age open clusters (NGC 2099, NGC 3114, NGC 6204, NGC 6705 and NGC 6756) via the narrow band $\Delta a$-system. The detection limit for photometric peculiarity is very low (always less than $0.009 \mathrm{mag}$ ) due to the high number of individual frames used (193 in total). We have detected six peculiar objects in NGC 6705 and NGC 6756 from which one in the latter is almost certainly an unreddened late type foreground star. The remaining five stars are probably cluster members and bona fide chemically peculiar objects (two are $\lambda$ Bootis type candidates). Furthermore, we have investigated NGC 3114, a cluster for which already photoelectric $\Delta a$-measurements exist. A comparison of the CCD and photoelectric values shows very good agreement. Again, the high capability of our CCD $\Delta a$-photometric system to sort out true peculiar objects together with additional measurements from broad or intermediate band photometry is demonstrated.
\end{abstract}

Key words. stars: chemically peculiar - stars: early-type - techniques: photometric - open clusters and associations: general

\section{Introduction}

In continuation of our previous four papers dedicated to the search for chemically peculiar objects via CCD $\Delta a$-photometry (Paunzen et al. 2002b), another five open clusters in our Milky Way have been investigated.

We present $\Delta a$-photometry for NGC 2099, NGC 3114, NGC 6204, NGC 6705 and NGC 6756 resulting in the detection of six peculiar objects from which one turned out to be a late type foreground object.

Send offprint requests to: E. Paunzen,

e-mail: Ernst.Paunzen@univie.ac.at

* Based on observations obtained at Complejo Astronómico el Leoncito (CASLEO), operated under the agreement between the Consejo Nacional de Investigaciones Científicas y Técnicas de la República Argentina and the National Universities of La Plata, Córdoba y San Juan; ESO-La Silla, UTSO-Las Campanas and L. Figl Observatory, Mt. Schöpfl (Austria).

$\star \star$ Figure 1 and Table 2 are only available in electronic form at http://www. edpsciences.org

$\star \star \star$ Member of Carrera del Investigador del Consejo Nacional de Investigaciones Científicas y Técnicas de la República Argentina and Visiting Astronomer at Complejo Astronómico El Leoncito operated under agreement between Consejo Nacional de Investigaciones Científicas y Técnicas de la República Argentina and the National Universities of La Plata, Córdoba y San Juan.
The data of NGC 3114 allowed us to compare photoelectric measurements to those of the CCD system showing excellent agreement.

We discuss the individual clusters and their peculiar objects including Johnson $U B V$ and Strömgren $u v b y$ photometry.

\section{Observations, reduction and results}

Observations of the five open clusters were performed with the Bochum $61 \mathrm{~cm}$ (ESO-La Silla), the Helen-Sawyer-Hogg $61 \mathrm{~cm}$ telescope (UTSO-Las Campanas Observatory), the $2.15 \mathrm{~m}$ telescope at the Complejo Astronómico el Leoncito (CASLEO) and the L. Figl Observatory (FOA) with the $150 \mathrm{~cm}$ telescope on Mt. Schöpfl (Austria) using the multimode instrument OEFOSC. The characteristic of the instruments and field-of-views can be found in Bayer et al. (2000) and Paunzen et al. (2002b). The observing log is listed in Table 1.

The basic CCD reductions and a point-spread-functionfitting were carried out within standard IRAF routines. The way of calculating the normality line, deriving the errors and calibration of our $y$ measurements, is the same as in previous works (Paunzen et al. 2002b).

Table 2 lists our observed open clusters and their characteristics from the literature (as listed in the corresponding sections). Furthermore, the number of observed stars, the number of individual frames (Table 1), the regression coefficients for all 
Table 1. Observing log.

\begin{tabular}{lcccccc}
\hline \hline Cluster & Site & Date & $\#_{N}$ & $\#_{g_{1}}$ & $\#_{g_{2}}$ & $\#_{y}$ \\
\hline NGC 2099 & FOA & 03.1998 & 1 & 2 & 2 & 2 \\
NGC 3114 & CASLEO & 11.1998 & 4 & 14 & 17 & 19 \\
NGC 6204 & CASLEO & 08.2001 & 2 & 20 & 15 & 20 \\
NGC 6705 & ESO & 05.1995 & 5 & 15 & 15 & 13 \\
NGC 6756 & ESO & 05.1995 & 4 & 11 & 13 & 11 \\
& UTSO & 05.1995 & 1 & 1 & 2 & 1 \\
\hline
\end{tabular}

transformations and normality lines as well as the $3 \sigma$ detection limits are given.

The finding charts (only available in the electronic form) of our open clusters are shown in Fig. 1. We have grouped the charts according to the telescopes used and the very different field of views.

Tables 4 to 8 of Paunzen et al. (2002b) with all data for the individual cluster stars are available in electronic form at the CDS via anonymous ftp to cdsarc.u-strasbg. fr (130.79.125.5), http://cdsweb.u-strasbg.fr/cgi-bin/ qcat? J/A+A/395/823 or upon request from the first author. These tables include the cross identification of objects from the literature, the observed $\left(g_{1}-y\right)$ and $a$ values with their corresponding errors, $V$ magnitudes, the $(B-V)$ or $(b-y)$ values from the literature, $\Delta a$-values derived from the normality lines of $\left(g_{1}-y\right),(B-V)$ or $(b-y)$, and the number of observations, respectively.

The diagnostic diagrams for all five open clusters are shown in Fig. 2. Furthermore, the normality lines and the confidence intervals corresponding to $99.9 \%$ are plotted.

All photometric data and limits are given in magnitudes.

\subsection{NGC 2099}

A comprehensive study of this open cluster is given by Nilakshi \& Sagar (2002). They included all relevant sources and data from the literature.

We have observed only the brightest members $(V>13)$ of the core region and find no photometric $\mathrm{CP}$ candidate. Although we have only six frames in total, the $\Delta a$-detection limit is very low (0.009) caused by the brightness of the observed objects.

The Johnson $U B V$ measurements of the literature as listed in Table 1 of Nilakshi \& Sagar (2002) were averaged in order to derive mean photometric mean values within this system. The main sequence and the region of the red giants are clearly visible in Fig. 2.

\subsection{NGC 3114}

This cluster was already observed photoelectrically in the $\Delta a$-system by Maitzen et al. (1988). They observed 127 stars of this cluster with $V>12$ and found six CP2 stars. We have used NGC 3114 to further test our new CCD photometric system and to expand the $V$-range of observed members (it is also the less distant open clusters investigated so far in the CCD $\Delta a$-system with 920 pc). Unfortunately, all six bona-fide CP stars are either outside our observed field of view or too bright for CCD measurements. Nevertheless, we have seven stars in common with the work of Maitzen et al. (1988) for which we can immediately check the observed $\Delta a$-values. Admitting that this is only a check for a null result in $\Delta a$ we notice a very good coincidence of both sources/techniques.

The CCD Johnson $U B V$ for this cluster were taken from Sagar \& Sharples (1991) and Carraro \& Patat (2001).

Figure 2 shows the $V$ versus $\left(g_{1}-y\right)$ diagram for NGC 3114 . The main sequence goes down to about 18 th magnitude with many non-members especially cooler than $\left(g_{1}-y\right)>-0.5$. We find no further photometric $\mathrm{CP}$ stars for this cluster within a detection limit of 0.008 . This is probably caused by the temperature range of the observed members. We find that $\left(g_{1}-y\right)=$ -0.69 corresponds to $(B-V)_{0}=0.44$ or a spectral type of about F5. Earlier than that, only 43 objects have been observed (all other stars are cooler). Since the incidence of CP stars significantly drops towards spectral types of A5, the incidence of late type peculiar stars is rather improbable (note that already six have been discovered before).

\subsection{NGC 6204}

NGC 6204 was often analyzed with its companion Hogg 22 which lies only 6' away (both are not associated with each other). The first investigation by Whiteoak (1963) was substantiated by Moffat \& Vogt (1973). The latter give an earliest spectral type of B8 and a distance of 810 pc. Kjeldsen \& Frandsen (1991) presented CCD Johnson $U B V$ photometry deriving a distance of $1200 \mathrm{pc}$ and an age of $200 \mathrm{Myr}$. Later, Forbes \& Short (1996) confirmed these results using appropriate isochrones.

Our observations cover the whole area of NGC 6204 as described in Kjeldsen \& Frandsen (1991) and Forbes \& Short (1996) spanning a magnitude range of about nine magnitudes. Although 55 frames were observed in total resulting in a very low detection limit of 0.007 , no photometric $\mathrm{CP}$ candidate was discovered.

\subsection{NGC 6705}

The apparent radius of NGC 6705 (M11) is $16^{\prime}$ which is significantly more than the field of view of the telescope $\left(3^{\prime} \times 4^{\prime}\right)$ used for this investigation. We have therefore observed only the innermost region. Kjeldsen \& Frandsen (1991), Brocato et al. (1993) and Sung et al. (1999) presented extensive studies based on CCD Johnson $U B V I$ photometry. Sung et al. (1999) found mass segregation based on 166.491 stars brighter than 21th magnitude (see their Fig. 3). The slope of the mass function increases systematically with increasing radius from the center.

We have checked the consistency of all published CCD photometric sources. The photographic values listed by Johnson et al. (1956) and Mathieu (1984) were not considered since such measurements are in generally not as accurate as CCD ones. We find a general trend of the data listed by Brocato et al. (1993) with respect to those of Kjeldsen \& Frandsen (1991) and Sung et al. (1999). The offset is about 0.70 for Johnson $V$, furthermore a slope of 0.74 for Johnson $(B-V)$ was found. The data of Brocato et al. (1993) were also checked 
Table 3. Stars of the investigated open clusters with peculiar $\Delta a$-values; No. 17 of NGC 6756 is most probably an unreddened foreground star.

\begin{tabular}{|c|c|c|c|c|c|c|c|c|c|c|c|c|c|c|}
\hline Cluster & $\mathrm{No}_{1}$ & $\mathrm{No}_{2}$ & $X$ & $Y$ & $V$ & $a$ & $\sigma$ & $\left(g_{1}-y\right)$ & $\sigma$ & $\Delta a$ & $(B-V)$ & $\Delta a$ & $(b-y)$ & $\Delta a$ \\
\hline \multirow[t]{3}{*}{ NGC 6705} & 51 & 7730 & 85.1 & 419.6 & 11.84 & 0.695 & 0.005 & -0.558 & 0.006 & +0.045 & +0.383 & +0.046 & & \\
\hline & 56 & 7777 & 91.2 & 429.9 & 13.18 & 0.586 & 0.010 & -0.571 & 0.005 & -0.059 & +0.384 & -0.063 & & \\
\hline & 215 & 8157 & 267.5 & 520.4 & 12.00 & 0.674 & 0.007 & -0.596 & 0.008 & +0.037 & +0.380 & +0.026 & & \\
\hline \multirow[t]{3}{*}{ NGC 6756} & (17) & 33 & 120.0 & 176.3 & 14.91 & 0.737 & 0.006 & -0.238 & 0.006 & +0.052 & & & +0.661 & +0.051 \\
\hline & 46 & 24 & 234.9 & 233.4 & 14.51 & 0.741 & 0.009 & -0.218 & 0.008 & +0.049 & & & +0.670 & +0.053 \\
\hline & 52 & 66 & 256.5 & 440.6 & 15.74 & 0.629 & 0.012 & -0.209 & 0.011 & -0.067 & & & +0.706 & -0.067 \\
\hline
\end{tabular}

Col. 1: cluster name;

Col. 2: notation sorted after $X$ and $Y$, respectively (Fig. 1);

Col. 3: notation according to Sung et al. (1999; NGC 6705), Delgado et al. (1997; NGC 6756);

Cols. 4, 5: $X$ and $Y$ coordinates in the finding charts (Fig. 1);

Col. 6: Johnson $V$ magnitude;

Cols. 7, 8: mean $a$-index and its standard deviation;

Cols. 9, 10: mean $\left(g_{1}-y\right)$ value and its standard deviation;

Col. 11: deviation from cluster line $a_{0}=a+b \cdot\left(g_{1}-y\right)$ using the corresponding constants as listed in Table 2;

Col. 12: $(B-V)$ from the literature;

Col. 13: deviation from cluster line $a_{0}=a+b \cdot(B-V)$ using the corresponding constants as listed in Table 2;

Col. 14: $(b-y)$ from Delgado et al. (1997);

Col. 15: deviation from cluster line $a_{0}=a+b \cdot(b-y)$ using the corresponding constants as listed in Table 2.

against the photographic ones by Johnson et al. (1956) and Mathieu (1984) yielding essentially the same results. The correlations of all other data sources result in offsets close to zero with a slope of one (within the error bars). For our further analysis we have used photometric mean values from Kjeldsen \& Frandsen (1991) and Sung et al. (1999).

From Fig. 2 we find three photometric CP candidates for NGC 6705. Two of them (No. 51 and 56; 7730 as well as 8157 according to Sung et al. 1999) have significant positive $\Delta a$-values whereas one $(56 ; 7777)$ exhibit a negative one. Unfortunately, none of these objects is included in the membership determination by McNamara et al. (1977). All three stars have $(B-V)_{0}$ of -0.05 and $(U-B)_{0}$ values from -0.07 to -0.17 resulting in a $Q$ value from -0.04 to -0.14 (Golay 1974) qualifying them as bona fide cluster members (Fig. 2). These values are typically for spectral types of $\mathrm{B} 8$ to $\mathrm{A} 0$ assuming a reddening of $E(B-V)=0.43$.

According to the results of Cowley et al. (1970) the photometric indices are in very good agreement for late $\mathrm{B}$ type $\mathrm{Si}$ or Cr stars. If we assume $\left(V_{0}-M_{V}\right)=11.55$ (Sung et al. 1999) then absolute magnitudes of +0.3 and +0.5 for No. 51 and 215 can be derived. Again, these absolute magnitudes are typical for CP stars in this temperature range (Grenier et al. 1981; Gómez et al. 1998).

Object No. 56 exhibits a significant negative $\Delta a$-value of -0.059 which is typical for a Be or a very extreme $\lambda$ Bootis star. An absolute magnitude of +1.6 would be still in the range of classical field $\lambda$ Bootis stars (Paunzen et al. 2002a). However, spectroscopic observations have to clarify the true nature of this object.

We also note that Johnson et al. (1956) classified one star (No. 7483; Sung et al. 1999) as B3p (Blue Straggler?). Our photometry results in a $\Delta a$-value of +0.003 which seems to rule out a peculiar nature. But we have to emphasize that magnetic
$\mathrm{CP}$ stars often show a variation of the $\Delta a$-value over their magnetic cycle. On the other hand, the error of the $a$-value itself (averaged over five different nights) is only 0.003 which might be another argument against chemically peculiarity.

\subsection{NGC 6756}

The only recent paper including NGC 6756 is that of Delgado et al. (1997). They presented CCD Strömgren uvby photometry of 368 stars in the area of this open cluster. It is the most distant program cluster $(3300 \mathrm{pc})$ which allowed us the cover the complete cluster area.

In total, three objects are photometrically peculiar: No. 17, 46 (33 and 24 according to Delgado et al. 1997, respectively; both with positive $\Delta a$-values) and No. 52 (66; negative one).

For the following calculations we have used $E(b-y)=0.73$ and $\left(V_{0}-M_{V}\right)=12.60$ as listed in Delgado et al. (1997).

The Strömgren $u v b y$ indices for No. 46 further strengthen its chemically peculiar nature. We find $(b-y)_{0}=-0.060,\left[m_{1}\right]=$ $0.105,\left[c_{1}\right]=0.671$ and $M_{V}=-0.42$ which are typical for a B8 Si star (Cameron 1966).

The values for the apparent peculiar object No. 17 are typical for an unreddened late type star (Crawford \& Mander 1966) and rule out chemical peculiarity: $(b-y)=0.661,\left[m_{1}\right]=0.502$ and $\left[c_{1}\right]=0.340$. This case shows the importance of additional photometric observations in a broad or intermediate band system.

Unfortunately, Delgado et al. (1997) have only measured $(b-y)$ for No. 52 making a final comment on its nature difficult. If it is a cluster member, then $(b-y)_{0}=-0.024$ and $M_{V}=$ +0.41 which is in the range of the $\lambda$ Bootis group. Again, further photometric or spectroscopic data are needed to shed more light on its nature. 

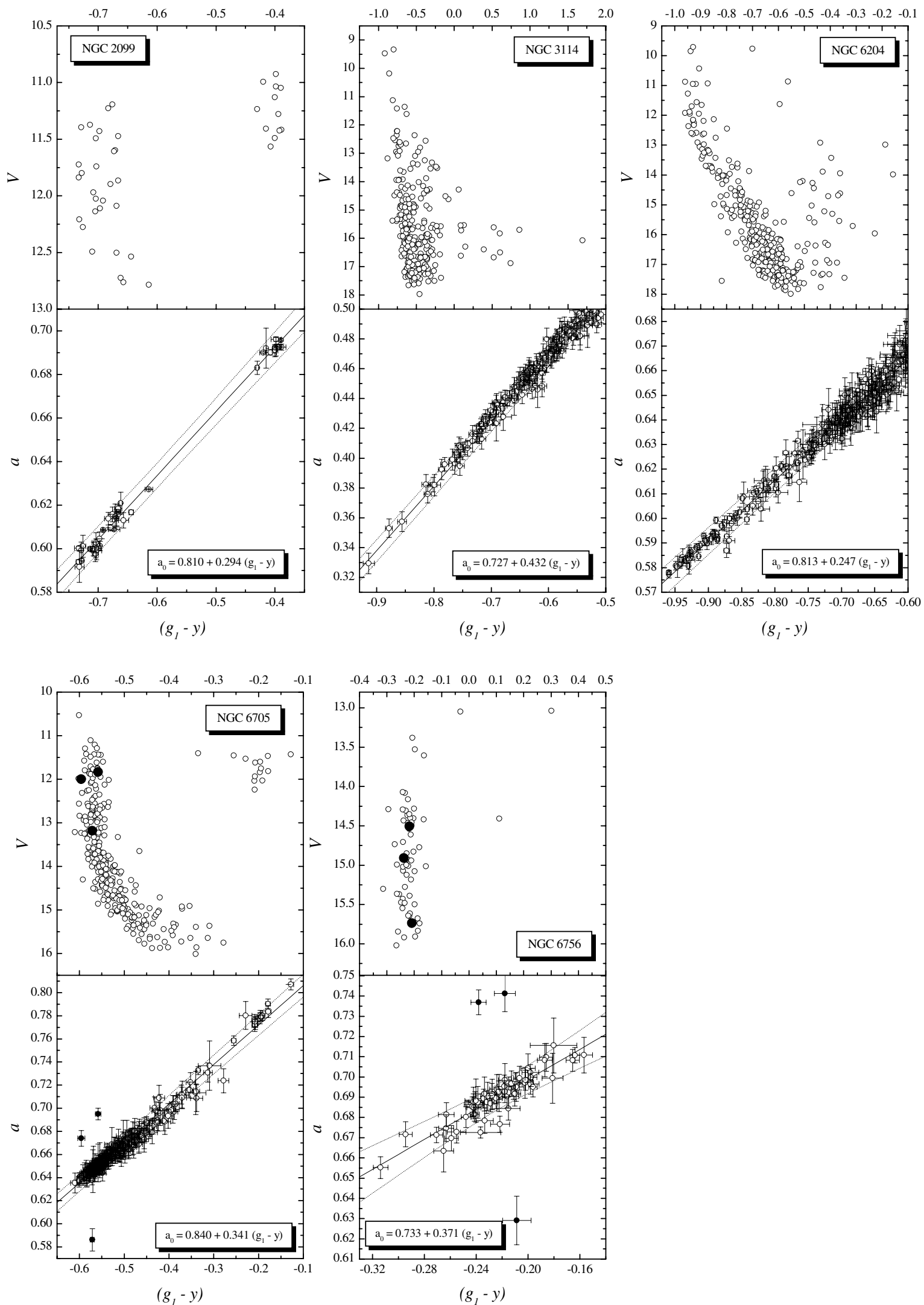

Fig. 2. Observed diagrams for our program clusters. The solid line is the normality line whereas the dotted lines are the confidence intervals corresponding to $99.9 \%$. The error bars for each individual object are the mean errors. The measurement errors of $V$ are much smaller than the symbols and have been omitted. The scales for the upper and lower diagrams of NGC 3114, NGC 6204 and NGC 6756 are different because the relevant range for peculiar objects in the $a$ versus $(g 1-y)$ diagrams seemed worthwhile to be shown. 


\section{Conclusions}

We have presented high precision narrow band $\Delta a$-photometry for 1008 objects from 193 individual frames for five intermediate aged open clusters of the Milky Way. With a very low detection limit of less equal than 0.009 , we find six peculiar objects in NGC 6705 and NGC 6756. One object in NGC 6756 turned out to be a late type foreground object. NGC 3114 served as further test case to compare the new CCD with the old photoelectric $\Delta a$-system. It has been shown that our narrow band $\mathrm{CCD} \Delta a$-photometric system together with additional measurements of the Johnson $U B V$ ot Strömgren $u v b y$ systems is highly efficient to detect peculiar objects on the upper main sequence.

Acknowledgements. The CCD and data acquisition system at CASLEO has been partly financed by R. M. Rich through U.S. NSF Grant AST-90-15827. This work benefitted from the financial contributions of the City of Vienna (Hochschuljubiläumsstiftung projects: Wiener Zweikanalphotometer and H-112/95 Image Processing). EP acknowledges partial support by the Fonds zur Förderung der wissenschaftlichen Forschung, project P14984. Use was made of the SIMBAD database, operated at CDS, Strasbourg, France and the WEBDA database, operated at the Institute of Astronomy of the University of Lausanne. This research has made use of NASA's Astrophysics Data System.

\section{References}

Bayer, C., Maitzen, H. M., Paunzen, E., Rode-Paunzen, M., \& Sperl, M. 2000, A\&AS, 147, 99
Brocato, E., Castellani, V., \& Digiorgio, A. 1993, AJ, 105, 2192

Cameron, R. C. 1966, Georgetown Obs. Monogram, 21

Carraro, G., \& Patat, F. 2001, A\&A, 379, 136

Cowley, A. P., Jaschek, M., \& Jaschek, C. 1970, AJ, 75, 939

Crawford, D. L., \& Mander, J. 1966, AJ, 71, 114

Delgado, A. J., Alfaro, E. J., \& Cabrera-Caño, J. 1997, AJ, 113, 713

Forbes, D., \& Short, S. 1996, AJ, 111, 1609

Gómez, A. E., Luri, X, Grenier, S., et al. 1998, A\&A, 336, 953

Golay, M. 1974, Introduction to astronomical photometry (Dordrecht: D. Reidel Publishing Co)

Grenier, S., Jaschek, M., Gómez, A. E., Jaschek, C., \& Heck, A. 1981, A\&A, 100, 24

Johnson, H. L., Sandage, A. R., \& Wahlquist, H. D. 1956, ApJ, 124, 81

Kjeldsen, H., \& Frandsen, S., 1991, A\&AS, 87, 119

Mathieu, R. D. 1984, ApJ, 284, 643

McNamara, B. J., Pratt, N. M., \& Sanders, W. L. 1977, A\&AS, 27, 117

Maitzen, H. M., Schneider, H., \& Weiss W. W. 1988, A\&AS, 75, 391

Moffat, A. F. J., \& Vogt, N. 1973, A\&AS, 10, 135

Nilakshi, \& Sagar, R. 2002, 381, 65

Paunzen, E., Iliev, I. Kh., Kamp, I., \& Barzova, I. 2002a, MNRAS, 336, 1030

Paunzen, E., Pintado, O. I., \& Maitzen, H. M. 2002b, A\&A, 395, 823

Sagar, R., \& Sharples, R. M. 1991, A\&AS, 88, 47

Sung, H., Bessell, M. S., Lee, H. W., Kang, Y. H., \& Lee, S. W. 1999, MNRAS, 310, 982

Whiteoak, J. B. 1963, MNRAS, 125, 105 
E. Paunzen et al.: Photometric search for peculiar stars in open clusters. V., Online Material p 1

\section{Online Material}


E. Paunzen et al.: Photometric search for peculiar stars in open clusters. V., Online Material p 2
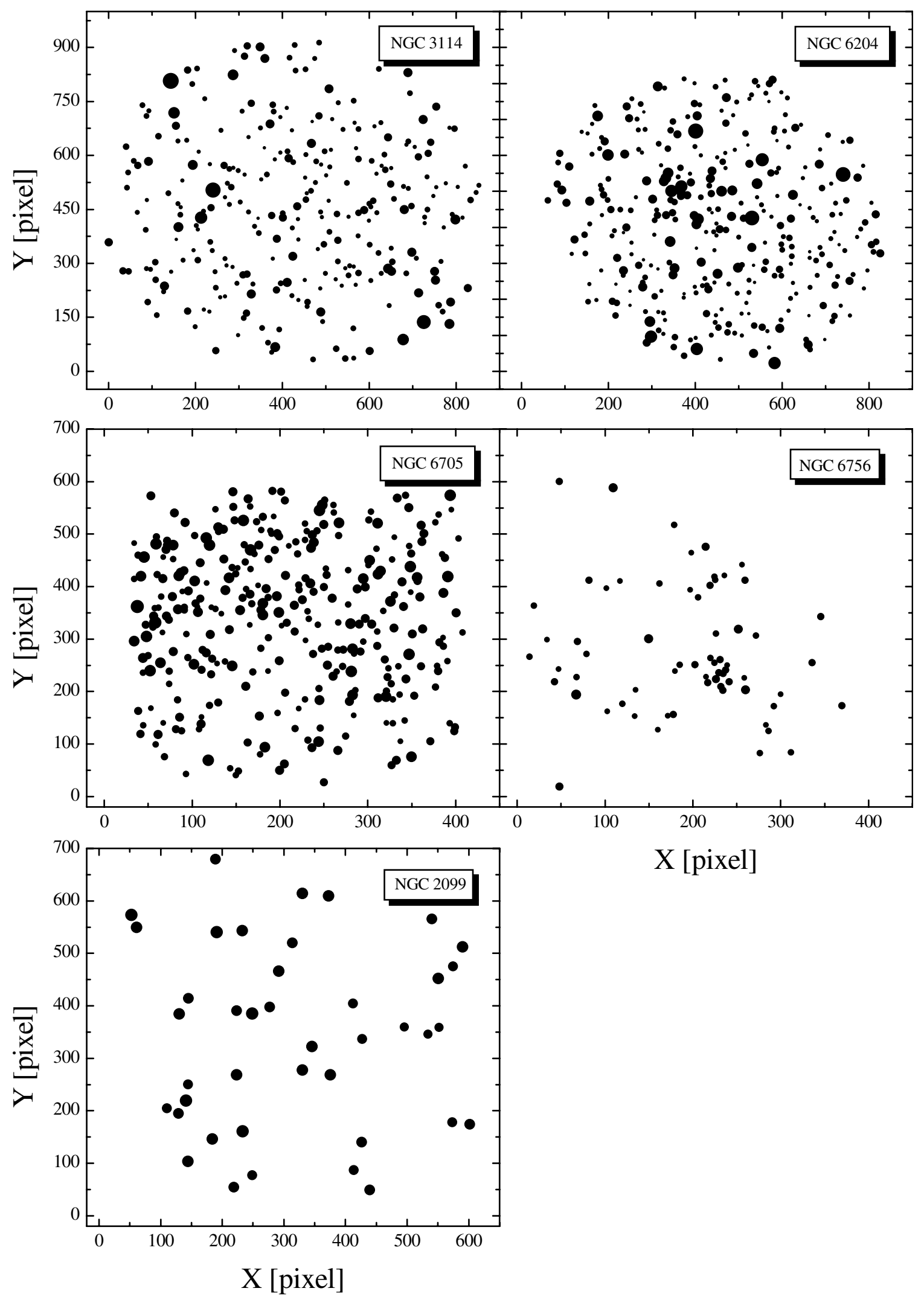

Fig. 1. Finding charts for the program clusters. North is to the right and west is upwards; 1 pixel $=0.5^{\prime \prime}$. The sizes (by area) of the open circles are inversely proportional to the $V$-magnitudes taken from Tables 4 to 8 . Larger open circles denote brighter objects. We have plotted the clusters sorted for the individual field of views. 
E. Paunzen et al.: Photometric search for peculiar stars in open clusters. V., Online Material p 3

Table 2. Summary of results and parameters from the literature. The errors in the final digits of the corresponding quantity are given in parenthesis.

\begin{tabular}{|c|c|c|c|c|c|}
\hline Name & $\begin{array}{c}\text { NGC 2099 } \\
\text { C0549+325 }\end{array}$ & $\begin{array}{c}\text { NGC 3114 } \\
\text { C1001-598 }\end{array}$ & $\begin{array}{c}\text { NGC 6204 } \\
\text { C1642-469 }\end{array}$ & $\begin{array}{c}\text { NGC 6705 } \\
\text { C1848-063 }\end{array}$ & $\begin{array}{c}\text { NGC 6756 } \\
\text { C1906+046 }\end{array}$ \\
\hline$l / b$ & $178 /+3$ & $283 /-4$ & $339 /-1$ & $27 /-3$ & $39 /-2$ \\
\hline$E(B-V)$ & 0.30 & 0.07 & 0.45 & 0.43 & 0.91 \\
\hline$d[\mathrm{pc}]$ & 1400 & 920 & 1200 & 1900 & 3300 \\
\hline $\log t$ & 8.60 & 8.48 & 8.30 & 8.40 & 8.11 \\
\hline Tr-type & II $1 \mathrm{r}$ & II $3 \mathrm{r}$ & I $3 \mathrm{~m}$ & I $2 \mathrm{r}$ & I $2 \mathrm{~m}$ \\
\hline $\mathrm{n}(\mathrm{obj})$ & 41 & 271 & 319 & 312 & 65 \\
\hline$\Delta a /(B-V)_{0}$ & & & & $\begin{array}{l}+0.046-0.047 \\
+0.026-0.046 \\
-0.063-0.050\end{array}$ & \\
\hline$\Delta a /(b-y)_{0}$ & & & & & $\begin{array}{l}+0.051-0.069 \\
+0.053-0.060 \\
-0.067-0.024\end{array}$ \\
\hline$V=a+b \cdot(y)$ & $-1.51(23) 1.01(2)$ & $-4.69(7) 1.008(3)$ & $-4.75(8) 0.987(4)$ & $-8.38(5) 1.009(2)$ & $-7.42(15) 0.962(7)$ \\
\hline$a_{0}=a+b \cdot\left(g_{1}-y\right)$ & $0.810(2) 0.294(4)$ & $0.727(1) 0.432(1)$ & $0.813(1) 0.247(2)$ & $0.840(3) 0.341(7)$ & $0.773(5) 0.371(22)$ \\
\hline$a_{0}=a+b \cdot(B-V)$ & $0.575(2) 0.097(3)$ & $0.353(6) 0.164(6)$ & $0.545(2) 0.103(2)$ & $0.602(1) 0.122(2)$ & \\
\hline$a_{0}=a+b \cdot(b-y)$ & & & & & $0.546(17) 0.212(25)$ \\
\hline $3 \sigma[\mathrm{mag}]$ & 0.009 & 0.008 & 0.007 & 0.008 & 0.009 \\
\hline$n$ (frames) & 6 & 50 & 55 & 43 & 39 \\
\hline
\end{tabular}

